\title{
Facilitating open-science with realistic fMRI simulation: validation and application
}

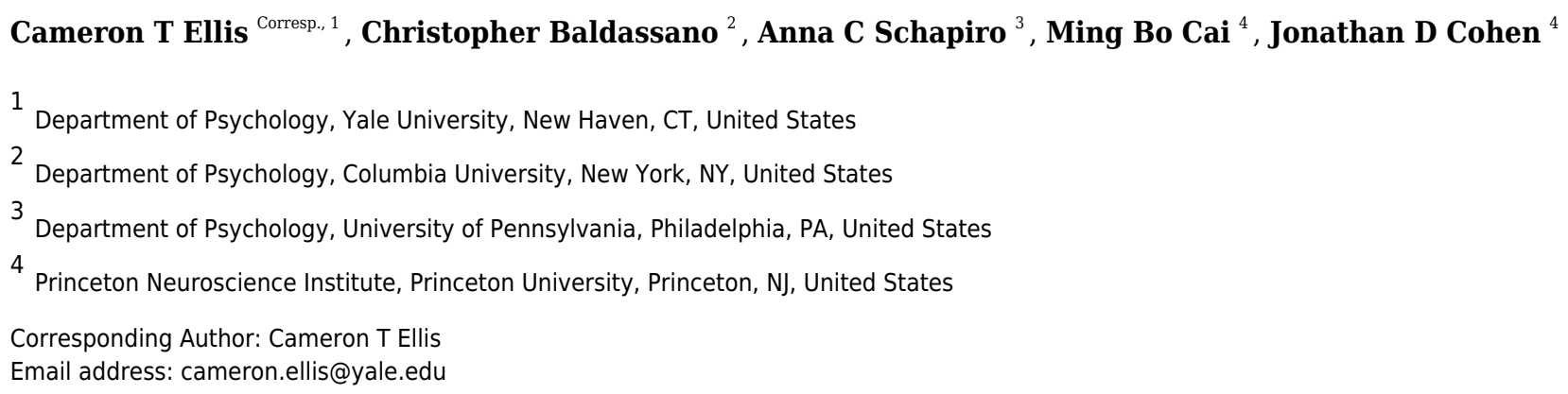

With advances in methods for collecting and analyzing fMRI data, there is a concurrent need to understand how to reliably evaluate and optimally use these methods. Simulations of fMRI data can aid in both the evaluation of complex designs and the analysis of data. We present fmrisim, a new Python package for standardized, realistic simulation of fMRI data. This package is part of BrainIAK: a recently released open-source Python toolbox for advanced neuroimaging analyses. We describe how to use fmrisim to extract noise properties from real fMRI data and then create a synthetic dataset with matched noise properties and a user-specified signal. We validate the noise generated by fmrisim to show that it can approximate the noise properties of real data. We further show how fmrisim can help researchers find the optimal design in terms of power. The fmrisim package holds promise for improving the design of fMRI experiments, which may facilitate both the preregistration of such experiments as well as the analysis of $\mathrm{fMRI}$ data. 
1 Facilitating open-science with realistic fMRI simulation: validation and application

2 Cameron T. Ellis ${ }^{1 *}$, Christopher Baldassano ${ }^{2}$, Anna C. Schapiro ${ }^{3}$,

3 Ming Bo Cai ${ }^{4}$, \& Jonathan D. Cohen ${ }^{4}$

4

$5 \quad{ }^{1}$ Department of Psychology, Yale University, New Haven, CT, USA

$6 \quad{ }^{2}$ Department of Psychology, Columbia University, New York, NY, USA

7 32Department of Psychology, University of Pennsylvania, Philadelphia, PA, USA

8 4Princeton Neuroscience Institute, Princeton University, Princeton, NJ, USA

9

10 Corresponding Author:

11 Cameron T. Ellis

122 Hillhouse Avenue, New Haven, CT, 06511, USA

13 Email address: cameron.ellis@yale.edu

16 Article type: Research article

17 Abstract: $166 / 500$ words

18 Main text: 7979 words

19 Display elements: 5

20 References: 52 


\section{Abstract}

24

With advances in methods for collecting and analyzing fMRI data, there is a concurrent need to

26 understand how to reliably evaluate and optimally use these methods. Simulations of fMRI data

27 can aid in both the evaluation of complex designs and the analysis of data. We present fmrisim, a

28 new Python package for standardized, realistic simulation of fMRI data. This package is part of

29 BrainIAK: a recently released open-source Python toolbox for advanced neuroimaging analyses.

30 We describe how to use fmrisim to extract noise properties from real fMRI data and then create a

31 synthetic dataset with matched noise properties and a user-specified signal. We validate the noise

32 generated by fmrisim to show that it can approximate the noise properties of real data. We

33 further show how fmrisim can help researchers find the optimal design in terms of power. The

34 fmrisim package holds promise for improving the design of fMRI experiments, which may

35 facilitate both the pre-registration of such experiments as well as the analysis of fMRI data. 36

37 Keywords: fMRI, simulation, Multivariate design, Reproducibility, Power analysis 
has exploded as a method for studying human brain function. In the early years of fMRI research, linear models were used to describe the voxel by voxel differences in activity between conditions (Friston et al., 1995). However, over time there has been a progressive shift toward methods that assess multivariate activity in order to probe representations and dynamics that are distributed throughout the brain (McIntosh, Bookstein, Haxby, \& Grady, 1996; Norman, Polyn, Detre, \& Haxby, 2006). These new methods have taken advantage of high-performance computing and advanced software packages to answer questions that would otherwise be difficult to test with univariate procedures alone (Cohen et al., 2017). However, this shift has not been accompanied by commensurate advances in our understanding of how fMRI noise influences these analyses. Here we describe fmrisim, a new tool to address this need that can help researchers evaluate and anticipate nuances that advanced neuroimaging analyses may introduce, and that can facilitate open science.

In the past, simulation has been a useful approach to addressing important issues regarding $\mathrm{fMRI}$ data. For example, simulations have been used to characterize the hemodynamic response function (HRF) in the analysis of event-related designs (Burock, Buckner, Woldorff, Rosen, \& Dale, 1998), as well as the spatial noise properties of fMRI data in cluster thresholding (Foreman et al., 1995; Ward, 2000) and assessing inter-subject variability (Erhardt, Allen, Wei,

57 Eichele, \& Calhoun, 2012). However, others have noted (Welvaert \& Rosseel, 2014) that the 58 procedures for constructing these simulations are often not standard across studies and/or not clearly described. One remedy to this situation is neuRosim — an extensively developed toolbox

60 in R that can be used to accurately simulate task-based activity, especially for univariate designs, 
61 in a standardized way across experiments (Welvaert, Durnez, Moerkerke, Verdoolaege, \&

62 Rosseel, 2011). Another tool is STANCE, a MATLAB package that takes real data as a baseline

63 and adds user-specified signal on top of it (Hill, Liu, Nutter, \& Mitra, 2017). simTB is another

64 package that can match the spatial noise properties of its simulation to real data, and is

65 particularly useful for group ICA simulations and connectivity analyses (Erhardt et al., 2012).

66 These tools provide different approaches to simulating univariate brain activity, however they

67 have been focused largely on univariate analyses. Similar tools are needed that address the effect

68 of noise in multivariate analyses.

69 Synergistic with their use in data analysis, simulations can also facilitate open science

70 and reproducibility (Simonsohn, Nelson, \& Simmons, 2014). Reproducibility is especially

71 problematic in fMRI research, where researchers have innumerable preprocessing choices that

72 can have important consequences for their results, but are not always carried out in a standard

73 and/or clearly described manner. Although many remedies to this problem have been proposed,

74 such as standardized preprocessing pipelines (Esteban et al., 2018), one potential solution is pre-

75 registration (Munafò et al., 2017). Using services such as those offered by the Open Science

76 Framework, researchers can specify in advance their design procedures and analysis pipeline and

77 embargo it until review. Current guidelines for reproducible science encourage the pre-

78 registration of both experimental design and analysis. This can be difficult for fMRI analyses:

79 because of their scope and complexity it is sometimes hard to anticipate all of the possible

80 concerns that otherwise may arise in analysis - especially those caused by noise — which often

81 invites post-hoc analyses. However, the failure to provide algorithmically precise analysis plans

82 in advance can inflate the risk of false discovery due to post-hoc exploration of different analysis

83 pipelines. 
data is collected, researchers can simulate expected experiment effects embedded within a realistic model of fMRI noise, and construct a pipeline for analyzing the results that takes this into account. This would not only ensure explicit specification of the analysis procedure, but could provide more accurate and reliable estimates of effect sizes that can be used for power calculations. The result would be that interested readers could observe for themselves exactly what the researcher hypothesized and what analyses were planned in advance. This is not to discourage post-hoc, exploratory analyses; rather, it would simply assist in demarcating what was predicted and what should be considered post-hoc analysis, so that the proper analyses and interpretations can be applied to each.

To be most effective, a simulator should be integrated with the tools used for experimental design and data analysis. While there are a number of packages used for standard fMRI analysis (e.g. FSL [Jenkinson, Beckmann, Behrens, Woolrich, \& Smith, 2012]), and others

97 for multivariate analysis (e.g. the Princeton MVPA toolbox [Detre, et al., 2006]), there has recently been a large scale migration to Python for scientific computing, where tools such as Nilearn (Abraham et al., 2014) and Nipype (Gorgolewski et al., 2011) make it an increasingly attractive environment for fMRI data analysis. Among these is BrainIAK (Brain Imaging

101 Analysis Kit, http://brainiak.org), a newly released open-source Python toolbox that supports 102 advanced neuroimaging analyses, such as multivariate pattern analysis (Norman, et al., 2006),

103 full-correlation matrix analysis (Wang, Cohen, Li, \& Turk-Browne, 2015), Inter-Subject

104 Functional Connectivity (Simony et al., 2016), Bayesian Representational Similarity Analysis 105 (Cai, Schuck, Pillow, \& Niv, 2019), Hierarchical Topographical Factor Analysis (Manning, et 106 al., 2018) and Shared Response Modeling (Chen et al., 2015). These methods are 
107 computationally intensive but have been optimized to exploit modern advances in high

108 performance cluster computing. Thus, this represents a potentially valuable environment for a

109 simulation package, and in particular one that addresses the impact of noise in these advanced,

110 multivariate analysis methods. Ideally, this should be customizable, have appropriate defaults,

111 and integrate seamlessly with existing tools for experiment design and analysis. Critically, it

112 must be able to generate realistic, 'brain-like' data in order for it to work in a pre-registration

113 pipeline.

114 In what follows we describe such a package — called fmrisim — that is a set of open-

115 source Python functions integrated into the BrainIAK toolbox (Kumar et al., 2019). This package

116 builds on the innovations of previous fMRI simulators, but focuses on its application to

117 advanced, multivariate neuroimaging methods. The goal is to make realistic simulations of brain

118 data that can be inserted into a standard fMRI analysis stream which makes use of multivariate

119 methods. To achieve this, fmrisim employs a linear model that estimates and combines known

120 sources of neural noise. Below we describe the steps necessary to generate a dataset appropriate

121 for multivariate analysis. We then describe analyses to assess the quality of the simulation.

122 Finally, we discuss applications of the simulator for evaluating the efficacy of experimental

123 design and facilitating reproducibility.

\section{Materials - fmrisim}

fmrisim generates some of the known sources of fMRI noise and combines them to create

127 simulations that share some of the properties of real fMRI data. Unlike other simulation tools,

128 such as POSSUM (Graham, Drobnjak, Zhang, 2016), fmrisim is not a physics-based model but

129 instead linearly combines a set of noise sources, inspired by biology and the physics of MRI, that 
130 are tuned in a data-driven fashion. We suggest that fmrisim is realistic in the sense that it

131 approximates some, albeit not all, of the known properties of fMRI data (like simTB and

132 STANCE), while also producing entirely novel volumetric data that can be preprocessed with the

133 same tools that are used in typical fMRI analysis (like neuRosim).

134 fmrisim is developed under an Apache 2.0 license, meaning that it is free for distribution

135 and modification for both commercial and non-commercial use. As part of the BrainIAK

136 environment, updates and contributions to the package must pass an extensive suite of tests in

137 order to validate that the changes do not compromise the quality of the tools.

138 The process of simulating participant data with fmrisim can be described in three steps:

139 (1) specifying parameters, (2) generating noise, and (3) generating signal to add to the noise.

140 Here we describe at a conceptual level what is needed to simulate a multivariate dataset with

141 noise properties matched to a raw dataset. We encourage interested readers to go through the

142 Jupyter Notebook in BrainIAK that demonstrates the relevant code to perform these steps

143 (github.com/brainiak/brainiak/blob/master/examples/utils/fmrisim_multivariate_example.ipynb).

144 The source code for fmrisim is extensively commented for accessibility.

145 2.1. Specify parameters

146 In order to simulate fMRI data using fmrisim, the signal, noise, and acquisition parameters

147 must be described. While all of these parameters can be defined by the user, some of these can

148 instead be derived from real, pre-existing fMRI data and used to simulate data with similar

149 attributes. These parameters, and which ones can be derived from real data, are described in the

150 following section. In addition to these parameters, the simulation also needs a template that

151 represents an approximate baseline of the fMRI signal to which noise can be added. This spatial

152 volume is generated in fmrisim by averaging each voxel across time (Welvaert, et al., 2011) and 
153 is used to create a binary mask of brain and non-brain voxels (Abraham, et al., 2014). The

154 template will also reflect any signal differences across the brain due to coil element strength.

155 This template can be based on a standardized template, provided by default with fmrisim, or can

156 be created from real fMRI data that was acquired by the user.

\section{2.2. Generate noise}

158 fmrisim can generate various types of realistic fMRI noise. Much of the noise modeling 159 was inspired by neuRosim (Welvaert, et al., 2011). In fmrisim, a single function receives the

160 specification of noise parameters and simulates whole brain data with noise properties

161 approximating those parameters. Figure 1 shows examples of the noise types generated by 162 fmrisim.

163 The noise of fMRI data is comprised of multiple components: drift; auto-

164 regressive/moving-average; physiological; task-related noise and system noise. Drift and system

165 noise are assumed to reflect machine-related noise and thus affect the entire field of view of the 166 acquisition. The remaining components are assumed to be specific to the brain and thus have a 167 smoothness component related to the smoothness of functional data. Using Gaussian random

168 fields of a certain Full-Width Half-Max (FWHM), we can determine how smooth the temporal

169 noise is across voxels (Chumbley \& Friston, 2009). This volume of spatial noise is masked to

170 only include brain voxels. Drift and system noise are added to all voxels in a volume.

171 To simulate drift, cosine basis functions of different phases are combined (Eq 1.), with

172 longer runs being comprised of more basis functions (Welvaert, et al., 2011). The first basis

173 function is a cosine basis function with a half cycle of the specified period. Each subsequent

174 basis function has a frequency that is a multiple of the first basis function (akin to fmriprep

175 [Esteban et al., 2018]), but their amplitudes diminish, such that $99 \%$ of the drift power is below 
176 the specified periodicity.

177

178

179

180

181

182

183

184

185

186

187

188

189

190

191

193

194

195

196

197

198

199

200

201

202

203

$$
\text { Equation } 1: \sum_{i=1}^{\frac{L}{A}} \cos \left(\mathrm{i} \pi_{L}^{t}+p_{i}\right) r^{i-1}
$$

where $L$ is the length in seconds of the run, $A$ is the volume acquisition time (AKA TR) in seconds, $i$ is the basis function counter, $t$ is the timestamp of each volume in seconds, $p_{i}$ is a random phase in radians for each iteration of $i, r$ is the proportion of drop off found by solving $0.99=\left(1-r^{\frac{2 L}{P}}\right) /\left(1-r^{\frac{2 L}{A}}\right)$ for $r$, where $P$ is the periodicity (default is 150s).

Auto-regressive/moving-average (ARMA) noise is generated by creating a sequence of volumes, with each volume being generated by combining Gaussian noise with a specified proportion of the previous volumes (Purdon \& Weisskoff, 1998). Precisely, the AR component specifies how much activity at each previous time point contributes to the activity at the current time point. The MA component does the same but for how much noise from previous time points contributes to the current time point (Eq 2.). Note that $\mathrm{AR}$ is used to model noise because it will reflect the BOLD activity in regions that do not contain task-related signal. fmrisim provides tools to insert task-related signal with appropriate AR dynamics (section 2.3).

$$
\text { Equation 2: } Y_{t}=X_{t}+\varphi Y_{t-1}+\omega X_{t-1}
$$

where $Y$ is the simulated noise at time point $t, X$ is the component of new 3dimensional Gaussian noise, $\varphi$ is the auto-regressive weight, and $\omega$ is the moving average weight.

Physiological noise is modeled by combining sine waves comprised of heart rate $(1.17 \mathrm{~Hz})$ and respiration rate $(0.2 \mathrm{~Hz})$ (Biswal, Deyoe, \& Hyde, 1996) with random phase. Finally, task-related noise is simulated by adding Gaussian or Rician noise to time points where there are events (determined by the design of the experiment). The ARMA, physiological and task-related noise components are mixed together according to user-defined parameters and then set to the appropriate magnitude of temporal variance (Eq 3.), derived from the Signal-to-FluctuationNoise Ratio (SFNR). The SFNR, also known as temporal signal-to-noise, reflects how much 
204 temporal variation there is in brain voxels relative to their mean activity (Friedman \& Glover,

205 2006). If the SFNR is high then the amount of temporal variation will be low and thus so will

206 these temporal noise components. Drift is weighted separately and added after this.

207

208

209

210

211

212

213

214

215

216

217

218

219

220

221

222

223

224

225

226

227

228

229

$$
\text { Equation 3: } W\left(w_{A R M A} v_{A R M A}+w_{\text {physio }} v_{\text {physio }}+w_{\text {task }} v_{\text {task }}\right)+w_{\text {drift }} v_{\text {drift }}
$$

where $W$ is the brain-specific noise that is determined by the standard deviation of the detrended temporal variability, averaged across the brain, $w$ is a weight from 0 to 1 set by the user or fit on the data ( $w$ for ARMA, physiology and task-based noise must sum to 1 ), and $v$ is a 4-dimensional volume of normalized noise.

The volume created by the combination of these noise components is then added with system noise. System noise results from heat-related motion in MRI scans (Bodurka, Ye, Petridou, Murphy, \& Bandettini, 2007). System noise is Rician (Gudbjartsson \& Patz, 1995); however, we have determined that the distribution of voxel intensity in non-brain regions (including the skull and eyes which are sensitive to $\mathrm{T} 2 *$ measurements) is also approximately a Rician distribution (e.g., the eyes have high MR values but the vast majority of voxels are near zero). As stated above, fmrisim creates a template of voxel activity (both brain and non-brain voxels) to which the simulated noise is added. This template also has non-brain voxels with a Rician distribution, so adding Rician noise to voxels with an already Rician distribution leads to inappropriately 'spiky' data. Instead, we have found that system noise is better approximated by Gaussian noise added to the template of average voxel activity. The magnitude of system noise is determined by both a spatial noise component, which depends on the Signal to Noise Ratio (SNR) value, and a temporal component of system noise, that depends on the SFNR.

All the noise parameters, like SNR, can be manually specified; however, it is also possible to extract some of these noise parameters directly from an fMRI dataset using a single automated function in fmrisim. Specifically, fmrisim utilizes tools for estimating the SNR, SFNR, FWHM and auto-regressive noise properties of real data. This real data can come from a 
230 sample of previously collected fMRI data that you believe will have similar attributes (e.g.,

231 acquisition, signal to noise quality, etc.) to the data you wish to simulate. To calculate the SNR,

232 fmrisim compares brain signal with non-brain spatial variance. To do this, the activity in brain

233 voxels is spatially averaged for the middle time point and divided by the standard deviation in

234 activity across non-brain voxels for that same middle time point (Triantafyllou et al., 2005). To

235 estimate SFNR, fmrisim divides each voxel's mean activity by the standard deviation of its

236 activity over time, after it has been detrended with a second order polynomial. This is done for

237 every brain voxel and then averaged to give an estimate of the global SFNR. The FWHM of a

238 volume is calculated by first computing variance in the $\mathrm{X}, \mathrm{Y}$, and $\mathrm{Z}$ dimensions, converting this

239 to FWHM for each dimension and then averaging both across each dimension and across a

240 sample of time points. Finally, fmrisim estimates ARMA using the statsmodels Python package

241 by assuming an AR order of 1 and an MA order of 1 (i.e. consider only the previous time point).

242 This is calculated independently for 100 voxels and then averaged to give the estimate for a 243 participant.

244 The noise estimates of real fMRI data provided by the fmrisim tools described above can

245 then be used to simulate data with equivalent noise parameters; however, there are some noise

246 properties that fmrisim does not estimate. This is because they are otherwise trivially dealt with

247 in preprocessing (e.g. drift), or they are impossible to retrieve from the raw data without knowing

248 the latent signal that generated the brain data (e.g. task noise) or without greater temporal

249 precision (e.g. physiological noise). These types of noise must be specified manually in order for

250 them to be used by fmrisim.

251 Importantly, estimating noise parameters from empirical data depends on assumptions

252 about the appropriate noise properties of fMRI data and how they interact. If the data is already 
253 preprocessed then this can interfere with the way noise properties ought to be inferred. For

254 instance, if all non-brain voxels have been masked, then these zeroed-out voxels will be an

255 inappropriate baseline for non-brain variability. In addition, because of the stochasticity of the

256 simulation, the estimation is not fully invertible: simulating a brain with a set of noise parameters

257 and then estimating the parameters from that simulated brain will recover ones that are similar,

258 but not necessarily identical, to those originally specified. To address this, and generate

259 simulations with noise characteristics as close to the ones specified as possible, fmrisim

260 iteratively simulates and checks the noise parameters until they fall within a specified tolerance

261 of what was specified. This is useful for matching noise parameters to a specific participant, not

262 just to typical human fMRI data. The fitting tolerance is by default set to within 5\% (i.e. the

263 difference between each real and simulated noise parameter is less than 5\%). This can be made

264 stricter by the user if desired; however there is inherent stochasticity in the noise generation

265 process, especially for ARMA and system noise, that makes it less likely to converge when the

266 tolerance is set lower. Once the noise parameters are estimated, they are then used to generate

267 time series data to which one or more hypothesized neural signals are added.

\section{2.3. Generate signal and add it to noise}

269 fmrisim has tools to simulate many different types of neural signal. These tools are useful

270 for generating signals intended to represent patterns of activation in specific regions of interest

271 (ROIs). However, custom scripts may be necessary for more complex signals, such as activity

272 coupling between regions for connectivity analyses. To design signal activity, it is necessary to

273 establish the predicted effect size, the ROI/spatial extent of the signal, and its time course. For

274 instance, the notebook describes a case in which two conditions evoke different patterns of

275 activity in the same set of voxels in the brain. This pattern does not manifest as a uniform change 
276 in voxel activity across the voxels containing signal (i.e. the mean evoked activity is not different

277 between conditions). Instead, each trial of a condition evokes an independent pattern across

278 voxels. This activity can then be convolved with the hemodynamic response in order to estimate

279 the predicted pattern of measured activity. fmrisim also makes it easy to calibrate the magnitude

280 of a signal using various metrics, such as percent signal change or contrast to noise ratio

281 (Poldrack, Mumford, \& Nichols, 2011; Welvaert \& Rosseel, 2013).

282 The final step is to combine, through addition, the noise volume with the signal volume to

283 create a brain that is ready for preprocessing and analysis.

2842.4 Expected background for fmrisim users

285 fmrisim was designed with the goal of being intuitive and user-friendly in order to support

286 the adoption of simulation in the design and analysis of fMRI experiments. We expect that users

287 who are Python novices will understand the provided notebooks that show how the simulator

288 works. In order to use the simulator, no specialized mathematical or statistical background is

289 required; although familiarity with fMRI analysis is expected. For users to design and simulate

290 their own experiments, they need only basic understanding of Python scripting in order to adapt

291 the provided examples. Such use of fmrisim has been validated as part of a university course

292 (Kumar et al., 2019), in which intermediate Python users were able to adapt the simulation code

293 provided in various ways. If users run into issues with fmrisim, they are encouraged to reach out

294 through the BrainIAK forum.

295 2.5. Discussion

296 Above we described how fmrisim generates realistic fMRI data. For more detail we point

297 readers to the documentation, and to the online notebook that demonstrates the implementation

298 of a simulation to see how the tool is actually used. fmrisim is intended to be flexible, allowing 
299 for the generation and specification of many different types of fMRI noise and signal. Of course,

300 the simulator is not perfect: a complete simulation would require an understanding of all of the

301 latent causal interactions in the brain and how these manifest in fMRI data - a goal that remains

302 the focus of the research enterprise itself.

303 fmrisim is limited in the scope of noise that it attempts to simulate. There are likely many

304 sources of noise that contribute to fMRI noise, but remain poorly understood or undetected.

305 Moreover, there are known types of noise that fmrisim does not attempt to model (e.g., higher

306 order structure inherent in baseline or resting state activity; Raichle et al., 2001) and fmrisim

307 applies noise in a linear manner despite what we know about the non-linear dynamics of the

308 brain. Adding additional noise structure in future versions of this package could make it possible

309 to simulate functional connectivity, as other simulation packages have done successfully (Allen

310 et al., 2014).

311 Motion has substantial effects on fMRI data quality but is not accounted for here. Other

312 simulations have modeled the effects of rigid body transformations (Allen, et al., 2014) and

313 motion between slice acquisitions (Jones, Bandettini, \& Birn, 2008), but these effects of motion

314 can be removed with linear motion correction software. The more pernicious effects of motion

315 are harder to simulate, such as the non-linear changes to T1 relaxation across planes (Friston,

316 Williams, Howard, Frackowiak, \& Turner, 1996), and are not addressed in this package. Other

317 global effects on fMRI signal, like sharp inhalation or breath holding (Power et al., 2017), are

318 also not modeled here but could be in future releases.

319 fmrisim is also limited in that assumes the distribution of noise is uniform throughout the

320 brain, as determined by a 3-dimensional Gaussian. This assumption is incorrect since different

321 types of noise have stronger effects in different parts of the brain. For instance, physiological 
322 noise has greater effects near major arteries and ventricles. It might be possible to introduce non-

323 uniform noise if we assumed a standard template (and fmrisim supports this currently) but to do

324 this at a participant-specific level would require extensive preprocessing to locate the regions of

325 a participant's brain that are sensitive to certain types of noise. Moreover, the slice acquisition

326 protocol, be it uni-band or multi-band, will introduce susceptibility to noise that may vary by

327 slice. This is not implemented explicitly in fmrisim; nonetheless, slice specific differences are 328 implicitly included in the brain templates.

329 These limitations of fmrisim prevent it from being a fully realistic simulation of fMRI

330 data; however, the use of fmrisim to simulate at least some of the known properties of fMRI data

331 holds promise for improving experimental design and data analysis, as illustrated in the examples

332 below.

333

\section{Experiment 1 - Validation of fmrisim}

To evaluate the validity of the simulator, we test whether various noise parameters of the simulated data match the noise parameters of real data. All the code for these analyses and plots is available online (https://github.com/CameronTEllis/fmrisim_validation_application.git).

\subsubsection{Materials and methods}

For these analyses we used the publicly available Corr_MVPA dataset (Bejjanki, Da

340 Silveira, Cohen, and Turk-Browne (2017):

$341 \mathrm{http}: / /$ arks.princeton.edu/ark:/88435/dsp01dn39x4181). This dataset contains two task runs, two

342 rest runs and an anatomical image for each of 17 participants. Participants provided written

343 consent in accordance with the institutional review board of Princeton University. This is

344 appropriate for our analyses because it is a typical sized dataset with no preprocessing. For this 
345 data set we only considered the rest runs. These were collected on a 3T scanner (Siemens Skyra)

346 with a 16 channel head coil and a $\mathrm{T} 2 *$ gradient-echo planar imaging sequence $(\mathrm{TR}=1.5 \mathrm{~s}$,

$347 \mathrm{TE}=28 \mathrm{~ms}$, flip angle $=64^{\mathrm{O}}$, matrix $=64 \times 64$, slices $=27$, resolution $\left.=3 \times 3 \times 3.5 \mathrm{~mm}\right)$.

348 We compared the noise properties of the real and simulated data to test the efficacy of

349 fmrisim. To do this, the noise properties of the participant data were extracted and then used to

350 create simulated data. We then estimated the noise properties of the simulated data, including

351 SNR, SFNR, auto-regression, and FWHM. The noise properties of the original data were then

352 compared with those measured in the simulated data in order to determine the fidelity of the

353 simulation. In other words, we tested whether the known noise properties utilized by the

354 simulator were similar to the noise properties estimated from the data generated by the simulator.

355 We used the default fitting procedure and created 10 simulations for the rest runs of each

356 participant in order to measure the reliability of each simulation.

357 3.1.2. Results

358 Figure 2 illustrates that the simulator can at least superficially approximate both the

359 spatial and temporal properties of real fMRI data. Figure $2 \mathrm{C}$ shows the averaged power spectra

360 for 1000 randomly sampled voxels after they have been high-pass filtered (100s cut-off, butter

361 filter). This suggests that the real and simulated voxels have similar spectral properties.

362 To evaluate whether fmrisim can accurately simulate brain data with specific noise

363 parameters, we compared the noise parameters estimated from the real and simulated data. The

364 ideal pattern of results is that there is no difference between the real and simulated data. Figure 3

365 shows boxplots of the noise parameters of the real and simulated data across participants.

366 Notably, the majority of simulated brains (across all participants, runs and resamples) are within

$3675 \%$ of the target parameter (proportion within 5\% for each noise component: SNR=98.5\%, 
$368 \mathrm{SFNR}=100 \%$, Auto-Regression=92.1\%, FWHM=100\%). Although advantageous, fitting is not

369 perfect because of the sequential nature of the fitting process (e.g. SNR is fit before

370 autoregressive noise) and the inherent randomness in the simulation.

371 Not depicted in Figure 3 is the variance in the estimated noise parameters on each

372 simulation. Although each simulation has inherent randomness, the variance in estimates is small

373 relative to the variance between individuals with different noise parameters (mean variance of

374 noise parameters extracted from simulations: SNR: $M_{V a r}=0.008$, SFNR: $M_{V a r}=1.428$, Auto-

375 Regression: $M_{V a r}=0.0006$, FWHM: $\left.M_{V a r}=0.0002\right)$. Execution times for this data set were

376 reasonable: the simulation with fitting took $278.3 \mathrm{~s}(\mathrm{SD}=39.8 \mathrm{~s}, \max =381.9 \mathrm{~s})$ on average to

377 complete for each participant/run on a single core of an Intel Xeon E5 processor (8 cores,

$3782.6 \mathrm{GHz})$.

379 3.1.3. Discussion

380 We evaluated how well fmrisim is able to recreate the noise properties of fMRI data and

381 showed that it can do so adequately. Critically, the noise parameter estimates were

382 participant/run specific, which means that fmrisim can simulate data with noise properties

383 matched to individual participants. In this sense, we claim that fmrisim is realistic; however,

384 there are likely numerous nuanced and non-linear noise properties of real data not captured by

385 fmrisim. Moreover, the simulations described above are necessarily incomplete, since known and 386 important sources of noise, such as physiological noise (Raj, Anderson, \& Gore, 2001), could not

387 be estimated from the data. Nonetheless, we believe fmrisim can simulate many of the critical

388 properties that are necessary for optimizing experiment design.

389 Importantly, the tests outlined above reflect a lower bound of what an fMRI simulator is

390 able to do. Future improvements to fmrisim or other simulators should capture more than just the 
391 descriptive statistics of noise in fMRI data but should also reflect the latent noise generation

392 properties of the brain. Moreover, future improvements should include realistic simulations of

393 motion artifacts and global signal changes. Nevertheless, fmrisim provides a useful advance in

394 the ability to evaluate how fMRI noise and signal interact, that can be used for experimental

395 design and analysis. We demonstrate such potential in the example application described below. consequences on statistical power can be complex and difficult to anticipate without appropriate tools. Here, we describe how fmrisim can be used to optimally design an experiment for maximizing statistical power.

Statistical power, in the context of null hypothesis testing, reflects the likelihood of getting a significant result from a sample, assuming that there is a real effect to be found.

Designing experiments with sufficient power is critical for conducting reliable research (Cohen, 1992). Power tests are important at the design stage for estimating how the number of participants or trials impacts the likelihood of reaching significance (Poldrack, et al., 2011). However, they can also be useful after data collection for determining the likelihood of a given result (Button et al., 2013).

Power tests are particularly important for fMRI research: data acquisition is expensive,

410 sample sizes are small, and effect sizes can be weak. Statistical power in fMRI research is

411 affected by the size and character of the signal to be measured (which can vary widely across

412 brain region; Gläscher, 2009; Desmond \& Glover, 2002), the number of participants that will be

413 studied, and the threshold for significance. Typically, power has been assessed in fMRI studies 
414 with reference to previous research with similar designs, using these to predict the effect size for

415 that design. However, in cases for which such information is not available (e.g., in novel designs

416 and/or populations), simulations provide a valuable alternative approach: by simulating a

417 hypothesized signal in the brain, it is possible to estimate how many trials, participants, etc., are

418 needed to pass a significance threshold for the analysis of interest.

419 One aspect of fMRI design for which power issues have been explored is in selecting the 420 inter-stimulus interval (ISI). To maximize power in task-based fMRI, it is considered best to

421 have slow designs in which events are spaced out to mitigate the overlap of the hemodynamic

422 response (Friston, Zarahn, Josephs, Henson, \& Dale, 1999). However, sometimes the only way

423 to evaluate a research question is to use faster event-related designs, in which events occur

424 within 10s of each other, either because a slower design would be impractically long or because

425 the cognitive process requires faster paced stimuli and/or responses. Although fast designs mean

426 the brain's slow hemodynamic response overlaps more across events, a shorter ISI can allow for

427 more trials (i.e. the efficiency is increased), which can increase the statistical power for both

428 univariate (Dale, 1999) and multivariate (Kriegeskorte, Mur, \& Bandettini, 2008) analyses. This

429 is especially true when the time between events is randomly jittered (Burock, Buckner, Woldorff,

430 Rosen, \& Dale, 1998), as different combinations of overlap can be sampled to improve

431 deconvolution. Moreover, if conditions are presented multiple times then it is considered a good

432 design choice to present them in a randomized order, since it will result in different overlaps

433 between evoked representations (Burock, et al., 1998). Thus, ISI can be a critical factor in

434 determining the effect size and power of the design.

435 Although longer ISIs with randomized event sequences are generally considered to

436 optimize power, most demonstrations of this have been limited to univariate analyses (although 
437 see Kriegeskorte, et al. (2008)). However, several factors could complicate, and potentially

438 invalidate generalization of these findings to multivariate analyses. For instance, calculating

439 efficiency and covariance are important and tractable within univariate analyses, in which

440 different voxels are treated as representing different stimulus conditions; however, doing so

441 becomes much more complex if an effect is multivariate - that is, a single voxel is involved in

442 some or all of the stimulus conditions.

443 To evaluate the effects of ISI and some related design choices on power, we used fmrisim

444 with data from a study by Schapiro, Rogers, Cordova, Turk-Browne, and Botvinick (2013).

445 Below, we first describe use of fmrisim to observe what signal magnitude is necessary to match

446 their result. We then show how different design choices can influence the expected effect size.

447 This provides an example not only of how fmrisim can be used to explore the power of

448 experimental designs, but also how this facilitates open science by making it easy for researchers

449 to pre-register experiment analyses and clearly demarcate planned versus post-hoc analyses. All

450 of the analyses described in what follows, as well as the necessary data to perform them, are

451 available online (https://github.com/CameronTEllis/fmrisim_validation_application.git).

452 4.1. Materials

453 Schapiro, et al. (2013) examined how event segmentation is achieved in the brain. Figure

4544 shows a graph representing the 15 stimuli participants saw in this study and the possible

455 transitions (grey) between stimuli during the learning phase of the experiment. In their fMRI

456 experiment, the stimuli were fractal-like images presented for $1 \mathrm{~s}$ with a 1, 3, or 5s ISI. Although

457 the assignment of images to graph nodes was random, the structured temporal transitions

458 between these stimuli were expected to change the image representations to reflect the graph

459 community structure (Fortunato, 2010). As participants observed a random walk through the 
460 possible transitions, the graph structure tends to produce long sequences of stimuli from within

461 one community before transitioning to another community. Transitions between communities

462 were only possible by passing through certain nodes on the graph. Behavioral evidence

463 suggested that over the course of exposure, participants come to represent the transitions between 464 communities as event boundaries.

465 Participants were scanned while observing the sequences of stimuli, and the imaging data

466 were used to examine the similarity structure in the neural representations of the stimuli.

467 Participants provided written consent in accordance with the institutional review board of

468 Princeton University. fMRI data were collected in a 3T scanner (Siemens Allegra) with a 16

469 channel head coil and a $\mathrm{T} 2 *$ gradient-echo planar imaging sequence $(\mathrm{TR}=2 \mathrm{~s}, \mathrm{TE}=30 \mathrm{~ms}$, flip

470 angle $=90^{\circ}$, matrix $=64 \times 64$, slices $=34$, resolution $=3 \times 3 \times 3 \mathrm{~mm}$, gap $=1 \mathrm{~mm}$ ). After participants had

47135 minutes of exposure to random walks on the graph outside the scanner, they viewed

472 alternations between random walks and Hamiltonian paths (i.e. every node visited exactly once)

473 in the scanner. One particular Hamiltonian path was chosen for each subject (e.g. the perimeter

474 around the dots in Figure 4) and was presented in clockwise and counterclockwise directions. In

475 order to avoid potentially confounding item repetition effects in the random walks, the first three

476 items of each Hamiltonian path were ignored and analyses were performed on the remaining 12

477 items in the Hamiltonian paths. Over five runs, 20 participants were presented with 25 sets of

478 these Hamiltonian walks. Each run was $\mathrm{Z}$ scored in time and the TRs corresponding to $4 \mathrm{~s}$ after

479 the stimulus onset were extracted and averaged to represent the brain's response to the stimulus.

480 A "searchlight" approach was used to test which, if any, voxels in the brain represented the

481 community structure. This involves iterating the same computation over a subset ("searchlight")

482 of voxels centered on different voxels in the brain. The searchlight was a spatio-temporal tensor 
483 of $3 \times 3 \times 3 \times 15$ voxels centered on every voxel in the brain. In each searchlight the correlation

484 of patterns of voxel activity was computed for all pairs of stimuli, and these were tested to

485 determine whether the correlation for stimuli belonging to the same community was higher than

486 that for stimuli belonging to different communities. A permutation test evaluated the robustness

487 of these metrics across participants. Some regions of the brain, including the inferior frontal

488 gyrus and superior temporal gyrus, reliably showed a greater correlation between stimuli within a

489 community compared to stimuli in different communities.

490 4.2. Methods

$491 \quad$ Five steps were performed to simulate the data from Schapiro, et al. (2013): generate a

492 template of average voxel activity; extract the noise parameters using fmrisim; represent the

493 timing of each stimulus onset; implement a region of interest mask of the significant voxels; and

494 characterize the signal. All of these were performed using the aforementioned dataset, and then

495 the nature and magnitude of the signal was manipulated as described below.

496 We embedded this signal in a region of interest (ROI), identified by Schapiro et al.,

497 (2013) spanning the left superior temporal gyrus, as well as the anterior temporal lobe and

498 inferior frontal gyrus (totaling 442 voxels). The simulated signal was generated with an event-

499 related design in which the different stimuli on the community structure graph (Figure 4)

500 occurred one after the other. Each stimulus evoked a positive pattern of activity across all the

501 voxels in the ROI. This pattern was determined by that stimulus's position in the high-

502 dimensional embedding of the community structure. In particular, we generated a 2-dimensional

503 graph of the community structure, then an orthonormal transformation was used to embed these

504 points in 442-dimensional space (matching the number of ROI voxels) in a way that preserved

505 Euclidean distance. This meant that each of these voxels' activity represented a dimension along 
506 which each event could vary and the pattern across the voxels determined the event's

507 representation. The specified response to every event for each voxel was convolved with the

508 double gamma HRF (Friston, et al., 1998). The time course of the events was determined by the

509 stimulus presentation timing during the Hamiltonian test phases.

510 There were two critical parameters determining the representation of the community

511 structure: magnitude and density. Magnitude reflects the neural response that each stimulus

512 presentation evokes and this determines the overall range of the coordinate values in the graph.

513 Specifically, percent signal change was used to determine the evoked response. Percent signal

514 change was scaled for each voxel by taking the mean of that voxel's noise time-series, dividing it

515 by one hundred to make it a proportion and then multiplying it by the percent signal change to

516 get the magnitude of the evoked response for that voxel. Since different stimuli evoke different

517 magnitudes of responses, the magnitude of evoked response specified by the above procedure is

518 used to scale the peak stimulus response (i.e. the coordinate furthest from 0 ) and all other

519 responses for that voxel are scaled in proportion to their response in the embedding.

520 Density is the amount of clustering within a community. A high-density representation

521 means that all of the stimuli within a community are similar to one another, reflecting strong

522 event segmentation. In the simulation, a density of 1 means that points within a community are

523 completely overlapping, whereas a density of 0 means that all transitions between stimuli result

524 in equal length edges on the graph (Figure 5A). Importantly, magnitude and density are

525 independent: a participant's representation of the communities can be perfectly separated (high

526 density) but the neural response evoked by each stimulus might be minimal (low magnitude),

527 meaning that the community structure is not measurable due to neural noise. Hence, the 
528 combination of both magnitude and density jointly contribute to the degree of community

529 structure that can be measured neurally.

530 We simulated fMRI data for pairs of magnitude and density parameter values to observe

531 how they compared to the real data. These were processed using the same analysis pipeline,

532 including the metric for calculating within vs. between community distance, used for the real

533 data to get a summary statistic of the degree of community structure in the ROI where a known

534 signal was added. We took the difference in the permuted t-test statistic for ROI voxels in the

535 real and simulated participants to get an estimate of simulated signal strength relative to the real

536 data.

537 In addition to re-creating the design of the experiment, we used fmrisim to manipulate

538 design parameters and examine their impact on effect size. As discussed above, a critical

539 parameter in event-related design is the ISI. We used fmrisim to explore the impact that this may

540 have had on the results. To do this, time was added between events while preserving the trial

541 jittering, ordering, and trial number (e.g. if the original participant experienced a 3s ISI between

542 events then in this simulation more time was inserted to space out these events, but otherwise

543 nothing else was changed); allowing new data to be simulated based on this time course. A

544 simulation with 0s added to the ISI was a direct simulation of Schapiro, et al. (2013). This

545 analysis was run 10 times (i.e. 10 sets of participants were generated for each level of ISI and

546 signal parameter) to estimate the variability of these summary statistics.

547 We also used fmrisim to examine how the stimulus sequence may have impacted the

548 results; that is, what would have happened if stimuli were not presented in the Hamilitonian path

549 as was done by Schapiro, et al. (2013), but rather presented in a random order, irrespective of

550 their community structure (note that a randomized order was undesirable in the experiment 
551 because it may have interfered with the learned transition probabilities). For this analysis, the

552 order of stimuli from each Hamiltonian walk was shuffled (such that the same stimuli could

553 never occur consecutively). According to evidence from randomized univariate designs (Dale,

554 1999) this should boost signal greatly.

555 Finally, although increasing the ISI may boost signal, it also increases the duration of the

556 experiment. To investigate this, we also examined the effect of limiting the number of trials per

557 run. For this analysis, we increased the ISI between events in a Hamiltonian walk, as before, but

558 cut-off the sequence of stimuli when the run ended for the real participants, and analyzed the

559 truncated dataset to determine the influence of this manipulation on power.

560 4.3. Results

561 The first aim of these analyses was to determine the range of percent signal change

562 required to replicate the results from Schapiro, et al. (2013). To do so, we compared the

563 summary statistic for real and simulated data for different pairs of signal magnitude and density

564 parameters. Figure 5B shows the absolute value of this difference, with low difference scores

565 indicating the simulation more accurately approximated the real test statistic compared to high

566 difference scores. When the simulated community structure is low density, the signal magnitude

567 required to approximate the test statistic of Schapiro, et al. (2013) is higher than the signal

568 magnitude required when the density is greater. This analysis shows that the necessary

569 magnitude is within the plausible range, regardless of community density. Hence, if this

570 simulation were done before data collection (using default noise parameters from other

571 participants), this analysis would show that this design, in terms of the specified participant

572 number, trial number and ISI, is sufficiently powered to identify community structure. Indeed, if 
573 this simulation were run many times it could estimate the likelihood of achieving a significant

574 result, akin to the use of a traditional power analysis for calculating the probability of success.

575 We chose three pairs of magnitude and density parameters that lie along the trough of

576 Figure 5B (i.e. parameters that minimize the difference in test statistic between the real and

577 simulated data) and varied the ISI to observe the effect it has on the test statistic, as shown in

578 Figure 5C. The peak test statistic is greatest with a minimum ISI of 5s. Critically, the test statistic

579 plateaus after this ISI, suggesting that a slower event-related design would confer no additional

580 benefit, especially given the cost of additional experimental time. This pattern is present

581 regardless of the signal density, although a denser signal structure has a higher test statistic.

582 Surprisingly, and counter to dogma conquering univariate analyses, randomizing the

583 order of test stimuli did not greatly change the test statistic. Figure 5D shows that when the

584 minimum ISI is 1s, as in Schapiro, et al. (2013), and the stimulus sequences are randomized, then

585 the test statistic is lower than when they are presented in a Hamilitonian order especially with

586 low density representations. In other words, randomizing the stimulus sequence would decrease

587 the test statistic. However, if the ISI for these randomized sequences is increased then the test

588 statistics are only slightly greater than the test statistic for a Hamiltonian sequence. Hence,

589 randomizing would only be beneficial for this experiment design if the ISI were long.

590 Although increasing ISI increases the signal when the experiment duration is potentially

591 limitless (Figure 5C and 5D), experiments are practically constrained in terms of how long a

592 session can run. Figure 5E shows that when the experiment duration is constrained to be the

593 same as Schapiro, et al. (2013), the test statistic in general decreases. Interestingly, if the density

594 is above zero then there is a slight gain by adding an ISI of $2 \mathrm{~s}$ but no such benefit exists when the

595 density is 0 . This suggests that given the time constraints of fMRI, the design chosen by 
596 Schapiro, et al. (2013) was efficient and nearly optimal. However, such foresight is not always

597 forthcoming as noted in the discussion below.

598 4.4. Discussion

599 The aim of the present analyses was to show how fmrisim can help researchers make

600 informed design choices, regarding factors such as ISI and stimulus ordering, to attain the

601 highest statistical power in their design as possible. Across the different signal parameters, the

602 same trends in effect size were found for different ISIs: the effect size increases as the ISI

603 increases up to 5s, after which it plateaus; however, this gain in effect size was greatest when the

604 signal was dense. Counter to typical assumptions in fast event-related designs (Burock, et al.,

605 1998), a randomized order of stimuli slightly reduced the effect size when the ISI was short and

606 only slightly helped when the ISI was longer. Of course, any increase in ISI needs to be weighed

607 against how this affects experiment duration and thus the number of allowable trials. Others have

608 shown that longer ISIs can help the effect size despite such limits (Friston, et al., 1999), whereas

609 for this design we observed that increasing ISI resulted in a diminished effect size (Kriegeskorte,

610 et al., 2008), although this was non-monotonic for some signal densities.

611 The analyses described above provide an example of how fmrisim can validate the

612 plausibility of an experimental analysis; however, fmrisim can also be used to show the

613 implausibility of an experiment/analysis. We have taken this approach in other work using

614 fmrisim to demonstrate what experimental design procedures can and cannot result in signal that

615 can be recovered using a particular analysis procedure — topological data analysis (Ellis,

616 Lesnick, Henselman-Petrusek, Keller, \& Cohen, 2019). Hence we believe that fmrisim can be

617 used to demarcate what experiments and analyses are and are not plausible. The example

618 outlined above also suggests ways in which simulation can be used for pre-registration. For 
619 example, consider designing a study similar to that of Schapiro, et al. (2013), but that required a

620 change in some parameter(s), such as ISI. The data generation and analysis procedures described

621 above could be used to evaluate the influence of this manipulation on the hypothesized effect,

622 which can then be pre-registered and made openly accessible both during review and after

623 publication. The hypothesized results and final analyses could also be compared to what was pre624 registered and be interpreted accordingly.

625 In sum, the kinds of simulations supported by fmrisim can be used to improve the power 626 of designs and facilitate open science practices in research.

627

628

\section{Conclusion}

629

We have presented a new package, fmrisim, for simulation of realistic fMRI data. To our

630

knowledge, fmrisim is the only package capable of taking in raw fMRI data and generating

synthetic fMRI data that is approximately matched in terms of some of the raw data's noise

632

properties. This software is open-source and updates are screened with rigorous tests. Code for

all of the analyses and plots included here can be found in online public repositories to allow

634

users to utilize and explore this package for their own research. It is particularly useful that

635

fmrisim is written in Python, a rapidly expanding platform for advanced neuroimaging analysis.

636 We show here that fmrisim can be profitably used to evaluate the effects of design and analysis

637 parameters and facilitate pre-registration of fMRI data. With the accelerating development of

638 advanced neuroimaging methods, fmrisim can help evaluate and compare these methods on a

639 standardized and openly accessible software platform that can facilitate both data analysis and

640 further methods development.

641 
642

643

644

645

646

647

648

649

650

651

652

653

654

655

656

657

658

659

660

661

662

663

664

665

666

667

668

669

670

671

672

673

674

675

676

677

678

679

680

681

682

683

684

685

686

References

Abraham, A., Pedregosa, F., Eickenberg, M., Gervais, P., Mueller, A., Kossaifi, J., . . . Varoquaux, G. (2014). Machine learning for neuroimaging with scikit-learn. Frontiers in Neuroinformatics, 8, 1-14.

Allen, E. A., Damaraju, E., Plis, S. M., Erhardt, E. B., Eichele, T., \& Calhoun, V. D. (2014). Tracking whole-brain connectivity dynamics in the resting state. Cerebral cortex, 24(3), 663-676.

Bejjanki, V. R., Da Silveira, R. A., Cohen, J. D., \& Turk-Browne, N. B. (2017). Noise correlations in the human brain and their impact on pattern classification. PLoS computational biology, 13(8), e1005674.

Biswal, B., Deyoe, E. A., \& Hyde, J. S. (1996). Reduction of physiological fluctuations in fMRI using digital filters. Magnetic Resonance in Medicine, 35(1), 107-113.

Bodurka, J., Ye, F., Petridou, N., Murphy, K., \& Bandettini, P. (2007). Mapping the MRI voxel volume in which thermal noise matches physiological noise-implications for fMRI. Neuroimage, 34(2), 542-549.

Burock, M. A., Buckner, R. L., Woldorff, M. G., Rosen, B. R., \& Dale, A. M. (1998). Randomized event-related experimental designs allow for extremely rapid presentation rates using functional MRI. Neuroreport, 9(16), 3735-3739.

Button, K. S., Ioannidis, J. P., Mokrysz, C., Nosek, B. A., Flint, J., Robinson, E. S., \& Munafò, M. R. (2013). Power failure: why small sample size undermines the reliability of neuroscience. Nature Reviews Neuroscience, 14(5), 365-376.

Cai, M. B., Schuck, N. W., Pillow, J. W., \& Niv, Y. (2019). Representational structure or task structure? Bias in neutral representational similarity analysis and a Bayesian method for reducing bias. PLoS computational biology, 15(5), e1006299.

Chen, P.-H. C., Chen, J., Yeshurun, Y., Hasson, U., Haxby, J., \& Ramadge, P. J. (2015). A reduced-dimension $f M R I$ shared response model. Paper presented at the Advances in Neural Information Processing Systems.

Chumbley, J. R., \& Friston, K. J. (2009). False discovery rate revisited: FDR and topological inference using Gaussian random fields. Neuroimage, 44(1), 62-70.

Cohen, J. (1992). Statistical power analysis. Current directions in psychological science, 1(3), 98-101.

Cohen, J. D., Daw, N., Engelhardt, B., Hasson, U., Li, K., Niv, Y., . . Turk-Browne, N. B. (2017). Computational approaches to fMRI analysis. Nature Neuroscience, 20(3), 304313.

Peerj reviewing PDF | (2019:10:42162:1:0:NEW 1 Jan 2020) 
687

688

689

690

691

692

693

694

695

696

697

698

699

700

701

702

703

704

705

706

707

708

709

710

711

712

713

714

715

716

717

718

719

720

721

722

723

724

725

726

727

728

729

730
Dale, A. M. (1999). Optimal experimental design for event-related fMRI. Human brain mapping, $8(2-3), 109-114$.

Desmond, J. E., \& Glover, G. H. (2002). Estimating sample size in functional MRI (fMRI) neuroimaging studies: statistical power analyses. Journal of Neuroscience Methods, 118(2), 115-128.

Detre, G., Polyn, S. M., Moore, C. D., Natu, V. S., Singer, B., Cohen, J. D., ... \& Norman, K. A. (2006, June). The multi-voxel pattern analysis (MVPA) toolbox. In Poster presented at the annual Meeting of the organization for human brain mapping.

Ellis, C. T., Lesnick, M., Henselman-Petrusek, G., Keller, B., \& Cohen, J. D. (2019). Feasibility of topological data analysis for event-related fMRI, Network Neuroscience 3(3), 1-12, doi: 10.1162/netn_a_00095

Erhardt, E. B., Allen, E. A., Wei, Y., Eichele, T., \& Calhoun, V. D. (2012). SimTB, a simulation toolbox for fMRI data under a model of spatiotemporal separability. Neuroimage, 59(4), 4160-4167.

Esteban, O., Markiewicz, C., Blair, R. W., Moodie, C., Isik, A. I., Aliaga, A. E., . . Snyder, M. (2018). FMRIPrep: a robust preprocessing pipeline for functional MRI. bioRxiv, 306951.

Fortunato, S. (2010). Community detection in graphs. Physics reports, 486(3-5), 75-174.

Friedman, L., \& Glover, G. H. (2006). Report on a multicenter fMRI quality assurance protocol. Journal of Magnetic Resonance Imaging, 23(6), 827-839.

Friston, K. J., Fletcher, P., Josephs, O., Holmes, A., Rugg, M., \& Turner, R. (1998). Eventrelated fMRI: characterizing differential responses. Neuroimage, 7(1), 30-40.

Friston, K. J., Holmes, A. P., Poline, J., Grasby, P., Williams, S., Frackowiak, R. S., \& Turner, R. (1995). Analysis of fMRI time-series revisited. Neuroimage, 2(1), 45-53.

Friston, K. J., Williams, S., Howard, R., Frackowiak, R. S., \& Turner, R. (1996). Movement-related effects in fMRI time-series. Magnetic resonance in medicine, 35(3), 346-355.

Friston, K. J., Zarahn, E., Josephs, O., Henson, R., \& Dale, A. M. (1999). Stochastic designs in event-related fMRI. Neuroimage, 10(5), 607-619.

Gläscher, J. (2009). Visualization of group inference data in functional neuroimaging. Neuroinformatics, 7(1), 73-82. 
731 Gorgolewski, K., Burns, C. D., Madison, C., Clark, D., Halchenko, Y. O., Waskom, M. L., \&

732

733

734

735

736

737

738

739

740

741

742

743

744

745

746

747

748

749

750

751

752

753

754

755

756

757

758

759

760

761

762

763

764

765

766

767

768

769

770

771

772

773

774

775

776

Ghosh, S. S. (2011). Nipype: a flexible, lightweight and extensible neuroimaging data processing framework in python. Frontiers in neuroinformatics, 5, 13.

Graham, M. S., Drobnjak, I., \& Zhang, H. (2016). Realistic simulation of artefacts in diffusion MRI for validating post-processing correction techniques. NeuroImage, 125, 1079-1094.

Gudbjartsson, H., \& Patz, S. (1995). The Rician distribution of noisy MRI data. Magnetic resonance in medicine, 34(6), 910-914.

Hill, J. E., Liu, X., Nutter, B., \& Mitra, S. (2017). A task-related and resting state realistic fMRI simulator for fMRI data validation. Paper presented at the Medical Imaging 2017: Image Processing.

Jenkinson, M., Beckmann, C. F., Behrens, T. E., Woolrich, M. W., \& Smith, S. M. (2012). FSL. Neuroimage, 62(2), 782-790.

Jones, T. B., Bandettini, P. A., \& Birn, R. M. (2008). Integration of motion correction and physiological noise regression in fMRI. Neuroimage, 42(2), 582-590.

Kay, K. N., Winawer, J., Mezer, A., \& Wandell, B. A. (2013). Compressive spatial summation in human visual cortex. Journal of neurophysiology, 110(2), 481-494.

Kriegeskorte, N., Mur, M., \& Bandettini, P. (2008). Representational similarity analysisconnecting the branches of systems neuroscience. Frontiers in systems neuroscience, 2.

Kumar, M., Ellis, C. T., Lu, Q., Zhang, H., Capota, M., Willke, T. L., Ramadge, P. J., TurkBrowne, N.B., Norman, K. (2019). BrainIAK tutorials: user-friendly learning materials for advanced fMRI analysis. https://doi.org/10.31219/osf.io/j4sbc

Manning, J. R., Zhu, X., Willke, T. L., Ranganath, R., Stachenfeld, K., Hasson, U., ... \& Norman, K. A. (2018). A probabilistic approach to discovering dynamic full-brain functional connectivity patterns. NeuroImage. 180: 243-252.

McIntosh, A., Bookstein, F., Haxby, J. V., \& Grady, C. (1996). Spatial pattern analysis of functional brain images using partial least squares. Neuroimage, 3(3), 143-157.

Munafò, M. R., Nosek, B. A., Bishop, D. V., Button, K. S., Chambers, C. D., du Sert, N. P., . . Ioannidis, J. P. (2017). A manifesto for reproducible science. Nature Human Behaviour, $1,0021$.

Norman, K. A., Polyn, S. M., Detre, G. J., \& Haxby, J. V. (2006). Beyond mind-reading: multivoxel pattern analysis of fMRI data. Trends in cognitive sciences, 10(9), 424-430.

Poldrack, R. A., Mumford, J. A., \& Nichols, T. E. (2011). Handbook of functional MRI data analysis. Cambridge University Press.

Peer) reviewing PDF | (2019:10:42162:1:0:NEW 1 Jan 2020) 
777

778

779

780

781

782

783

784

785

786

787

788

789

790

791

792

793

794

795

796

797

798

799

800

801

802

803

804

805

806

807

808

809

810

811

812

813

814

815

816

817

818

819

820

821

822

Power, J. D., Plitt, M., Laumann, T. O., \& Martin, A. (2017). Sources and implications of wholebrain fMRI signals in humans. Neuroimage, 146, 609-625.

Purdon, P. L., \& Weisskoff, R. M. (1998). Effect of temporal autocorrelation due to physiological noise and stimulus paradigm on voxel-level false-positive rates in fMRI. Human brain mapping, 6(4), 239-249.

Raichle, M. E., MacLeod, A. M., Snyder, A. Z., Powers, W. J., Gusnard, D. A., \& Shulman, G. L. (2001). A default mode of brain function. Proceedings of the National Academy of Sciences, 98(2), 676-682.

Raj, D., Anderson, A. W., \& Gore, J. C. (2001). Respiratory effects in human functional magnetic resonance imaging due to bulk susceptibility changes. Physics in medicine and biology, 46(12), 3331.

Schapiro, A. C., Rogers, T. T., Cordova, N. I., Turk-Browne, N. B., \& Botvinick, M. M. (2013). Neural representations of events arise from temporal community structure. Nature neuroscience, 16(4), 486-492.

Simonsohn, U., Nelson, L. D., \& Simmons, J. P. (2014). P-curve: a key to the file-drawer. Journal of Experimental Psychology: General, 143(2), 534.

Simony, E., Honey, C. J., Chen, J., Lositsky, O., Yeshurun, Y., Wiesel, A., \& Hasson, U. (2016). Dynamic reconfiguration of the default mode network during narrative comprehension. Nature communications, 7, 12141.

Triantafyllou, C., Hoge, R., Krueger, G., Wiggins, C., Potthast, A., Wiggins, G., \& Wald, L. (2005). Comparison of physiological noise at $1.5 \mathrm{~T}, 3 \mathrm{~T}$ and $7 \mathrm{~T}$ and optimization of fMRI acquisition parameters. Neuroimage, 26(1), 243-250.

Wang, Y., Cohen, J. D., Li, K., \& Turk-Browne, N. B. (2015). Full correlation matrix analysis (FCMA): An unbiased method for task-related functional connectivity. Journal of Neuroscience Methods, 251, 108-119.

Ward, B.D. (2000). Simultaneous inference for fMRI data. Retrieved December 15, 2018, from http://homepage.usask.ca/ ges125/fMRI/AFNIdoc/AlphaSim.pdf

Welvaert, M., Durnez, J., Moerkerke, B., Verdoolaege, G., \& Rosseel, Y. (2011). neuRosim: An R package for generating fMRI data. Journal of Statistical Software, 44(10), 1-18.

Welvaert, M., \& Rosseel, Y. (2013). On the definition of signal-to-noise ratio and contrast-tonoise ratio for fMRI data. PloS one, 8(11), e77089.

Welvaert, M., \& Rosseel, Y. (2014). A review of fMRI simulation studies. PloS one, 9(7), e101953. 


\section{Figure 1}

Figure 1. Example time courses of the different types of noise that are generated by fmrisim.

Each plot represents a voxel's activity for each type of noise.

Drift
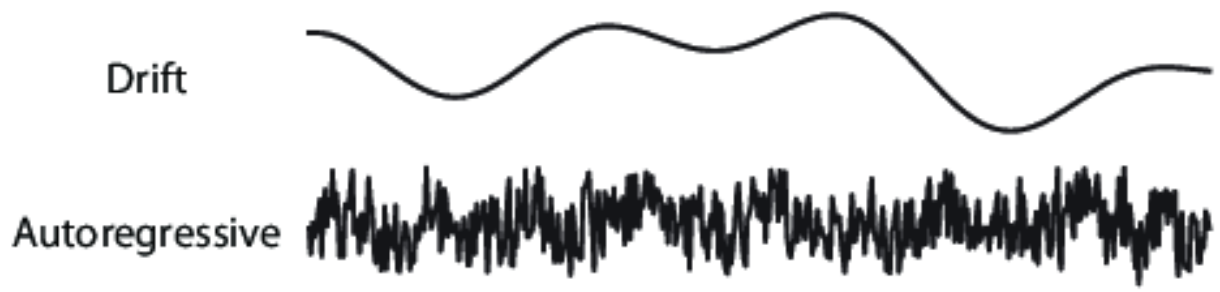

Physiological

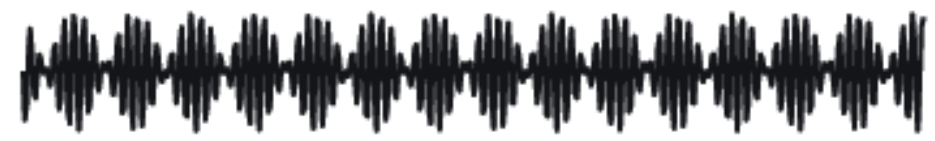

Task

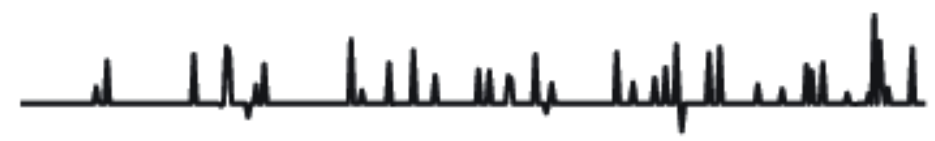

System

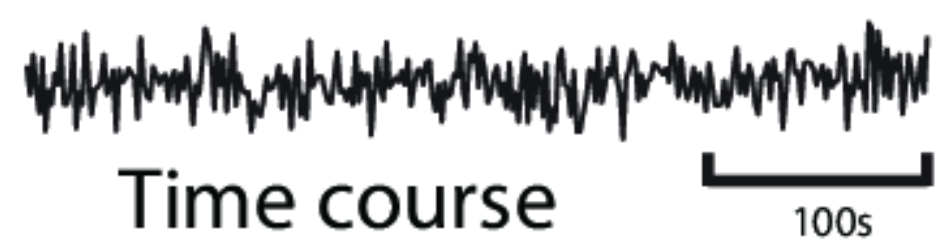


Figure 2

Figure 2. Example of the spatial and temporal structure of real and simulated data

(A) depicts the spatial structure of real data (top) and fitted simulated data (bottom). (B) shows the time course of sample voxels and (C) shows the power spectra of a sample of high-pass filtered voxels.

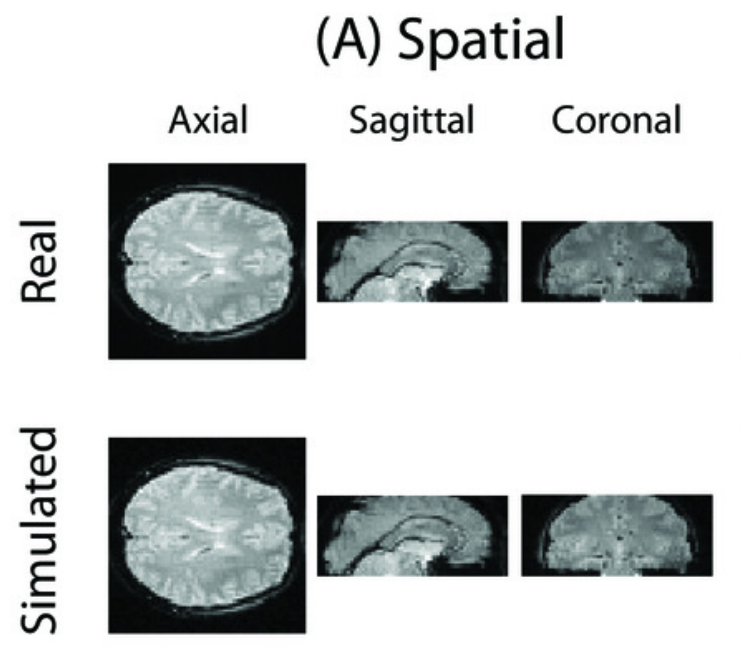

(B) Temporal

Voxel 1

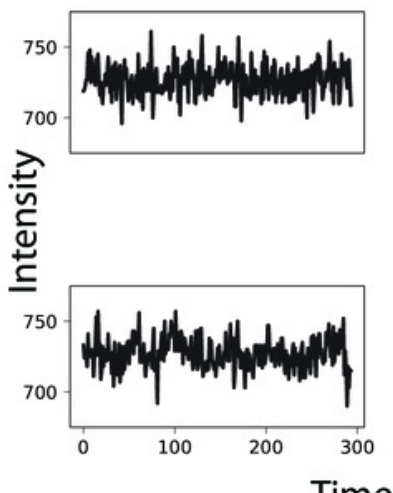

Voxel 2
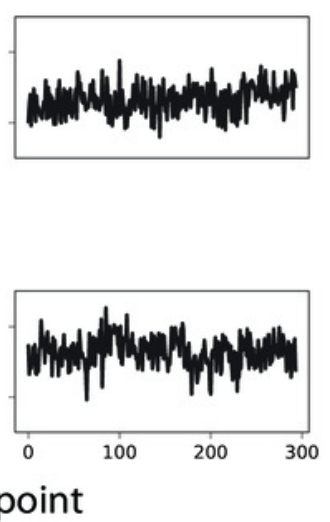

(C) Spectral

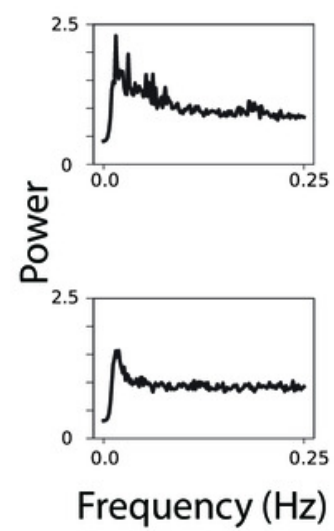




\section{Figure 3}

Figure 3. Boxplots of noise parameters from real data and fitted simulated data.

For each participant and run, 10 simulations of their real data were generated and their noise parameters estimated. These noise parameters were then averaged within participant/run and plotted, and compared with the noise parameters from their corresponding real data. The grey lines connect the participant/run parameter value, the ellipses refer to outliers. The noise parameters tested were (A) Signal-to-Noise Ratio, (B) Signal-to-Fluctuation-Noise Ratio, (C) Auto-Regression, (D) Full-Width Half-Max. 
(A)

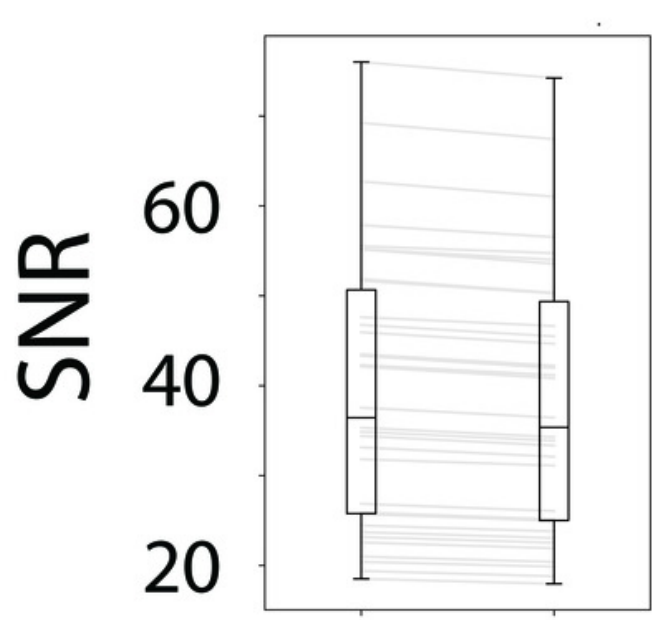

(B)

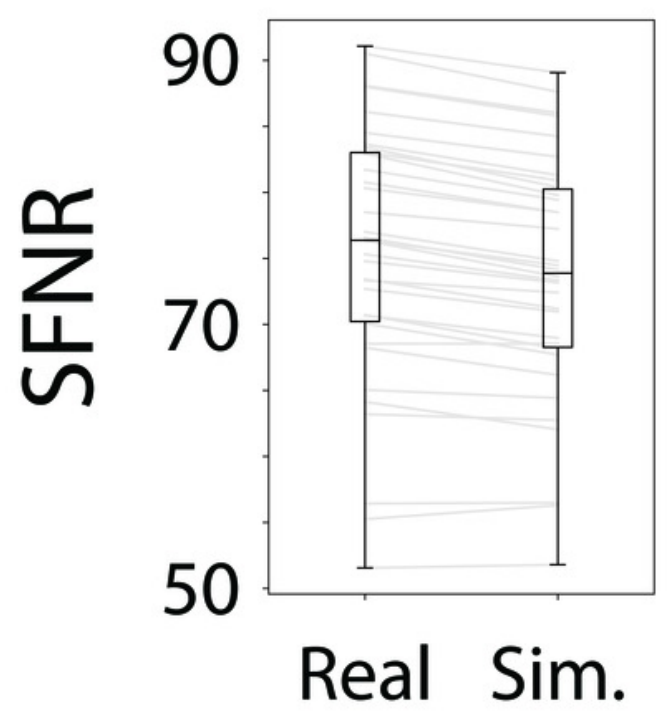

(C)

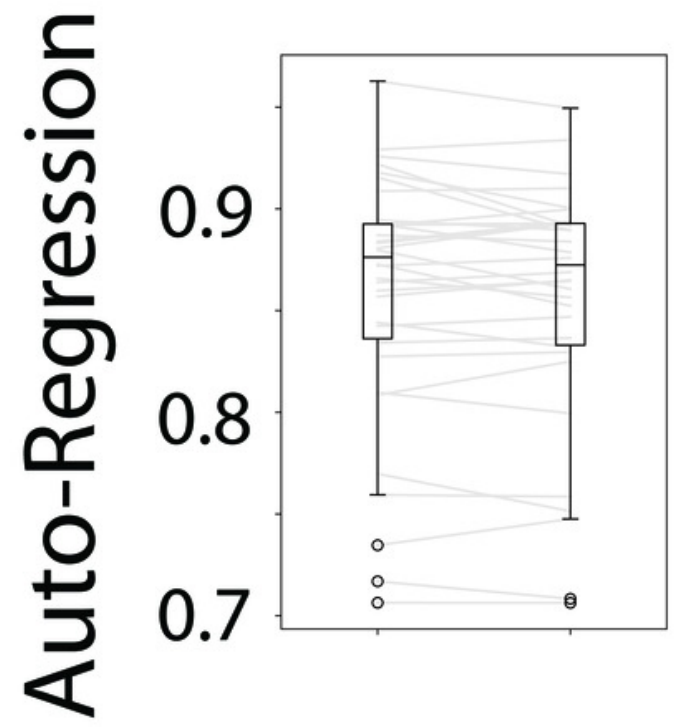

(D)

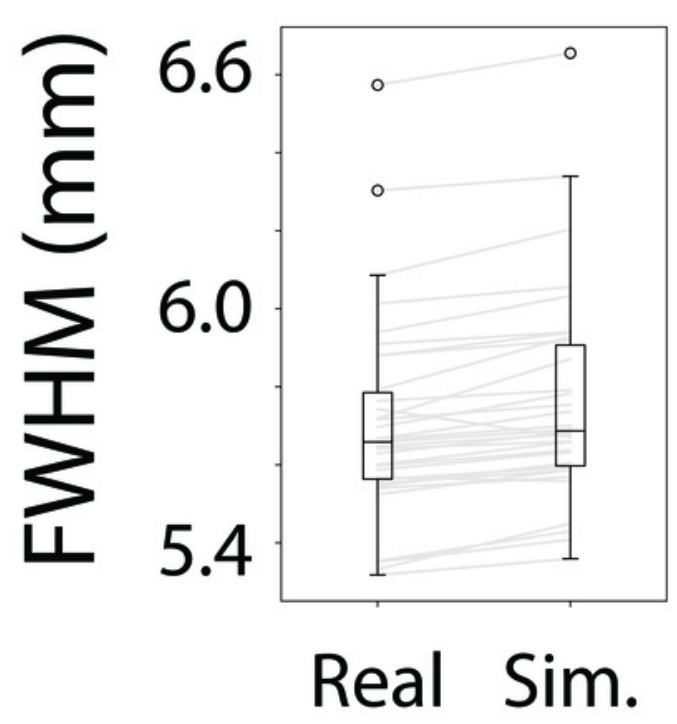


Figure 4

Figure 4. Graph of the community structure from Schapiro, et al. (2013).

Black dots represent the 15 abstract visual stimuli participants were shown. Grey lines represent possible transitions between the dots.

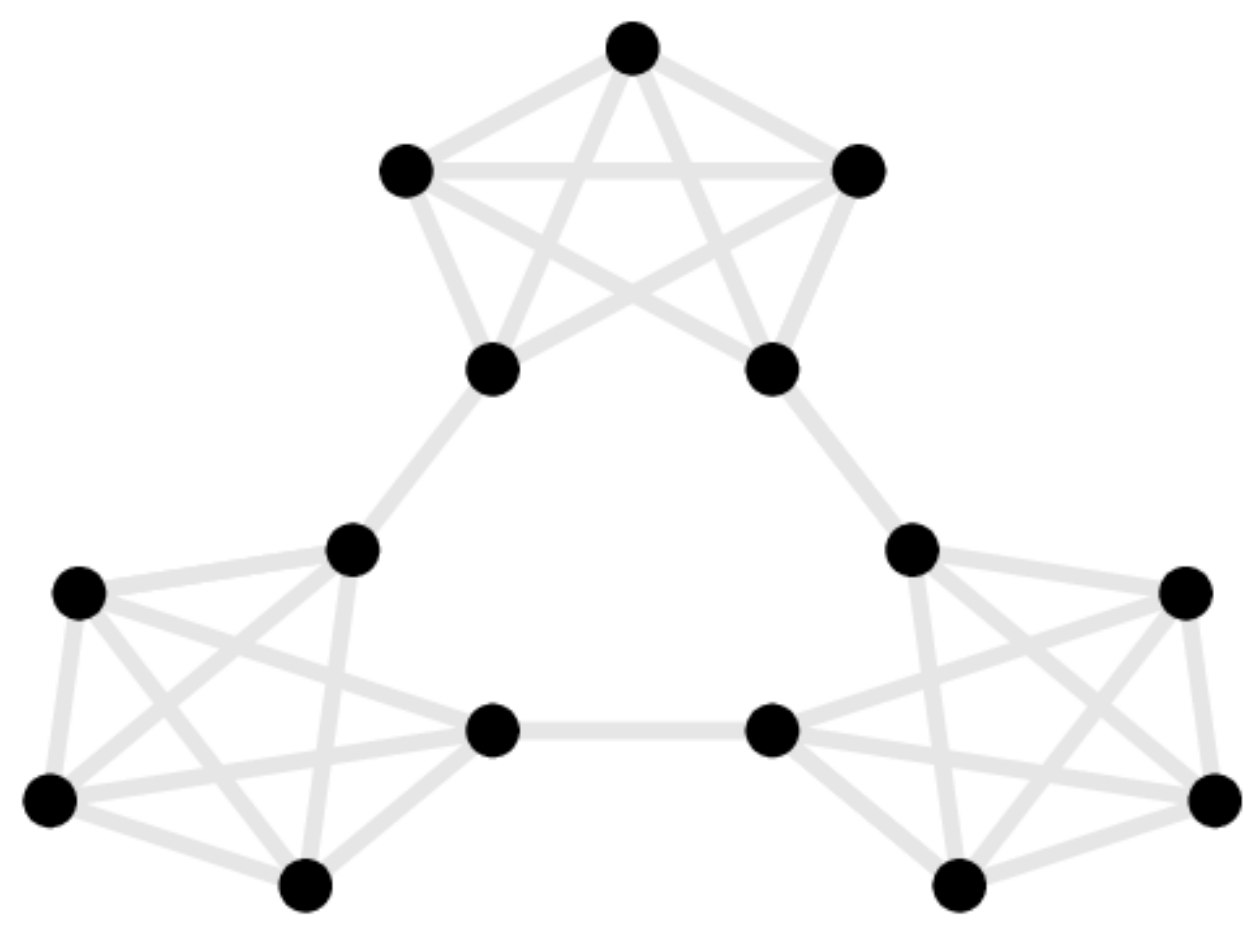




\section{Figure 5}

Figure 5. Alternative designs for Schapiro, et al. (2013) and their relative test statistic.

(A) Depictions of representations corresponding to different densities. (B) A heat map of the difference between the real data and simulated data for different parameters of signal magnitude and density. Three pairs of parameters were chosen that minimize the difference between the simulated and real data. (C) For these three pairs of parameters, the average tstatistic in the ROI that was significant in Schapiro, et al. (2013) was subtracted from the real data and compared at different ISIs when the stimuli were presented in a Hamiltonian sequence. The black line represents the real data. (D) is the same as (C) except that the sequence of stimuli was randomized in the simulation. (E) is the same as (C) except that the maximum duration of the run is limited, regardless of ISI. Shaded lines represent the standard deviation across the 10 permutations of this condition. ' $D$ ' in the legend refers to the density of the representation, ' $\%$ ' refers to the percent signal change. 
(A) Density
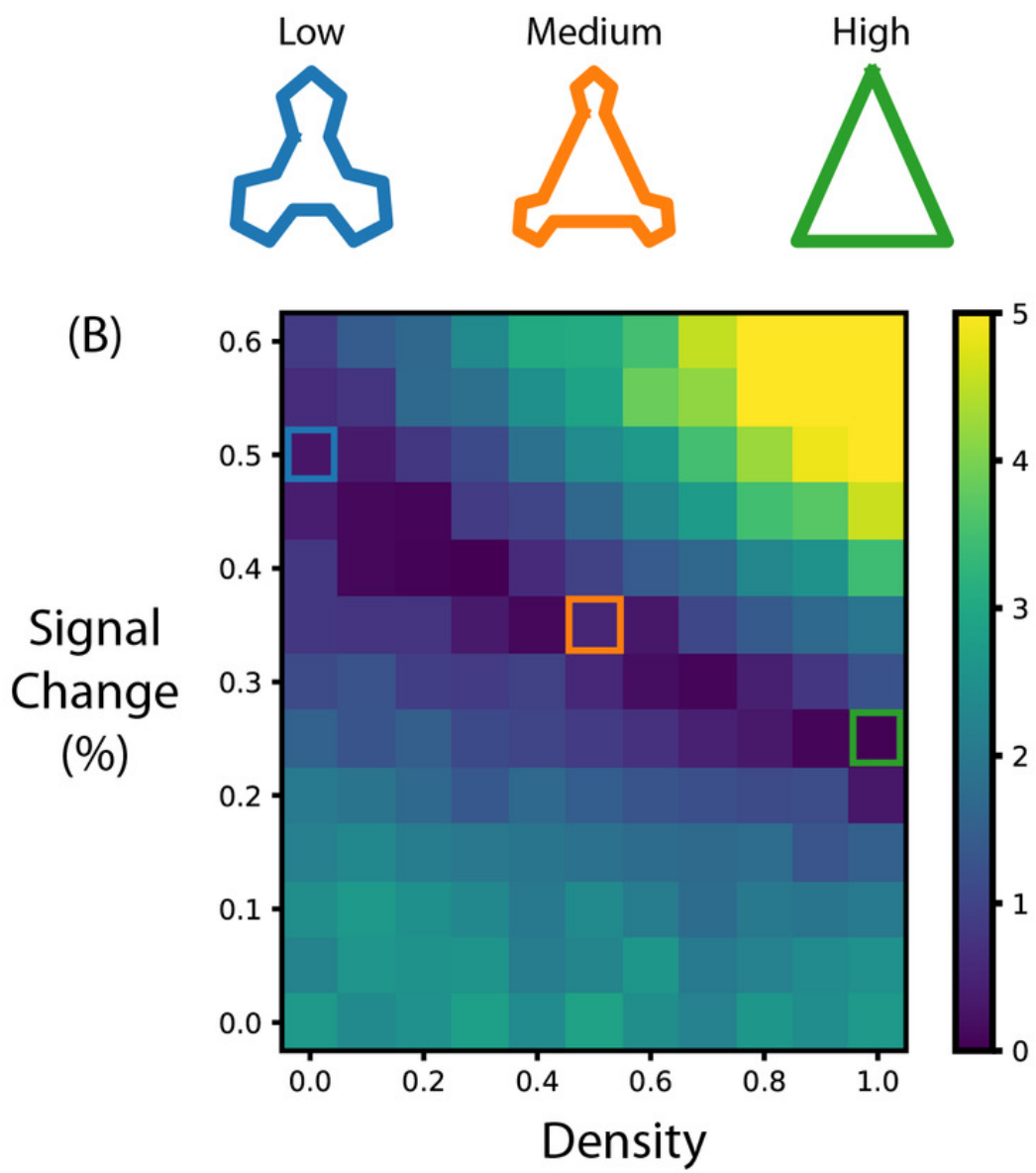

Real - Simulated (t stat, absolute)

(C) Hamiltonian

(D) Randomized

(E) Limited
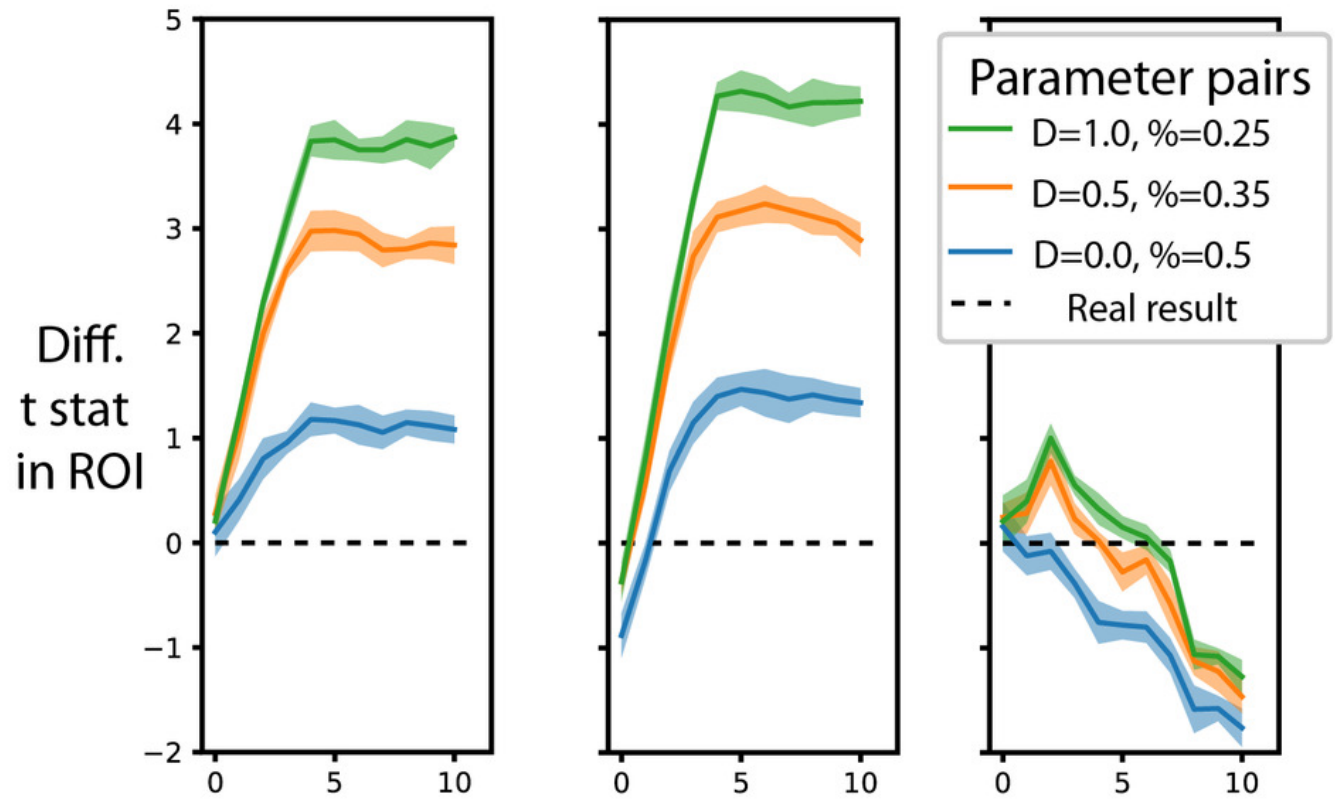

Added ISI (s) 